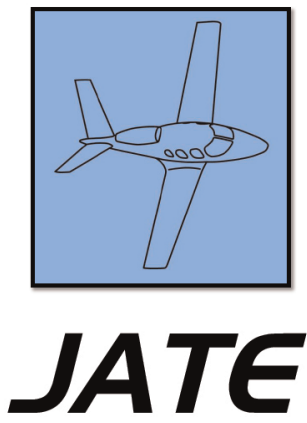

Journal of Aviation Technology and Engineering 2:2 (2013) 32-44

\title{
Comparison of Best Economy and Best Power Mixture Settings in the Purdue University Training Fleet Revisited
}

\author{
Ronald F. Brender and Thomas Q. Carney \\ Purdue University
}

\begin{abstract}
Best economy (lean of peak or LOP) operation of aircraft engines rather than best power (rich of peak or ROP) has been suggested as a means to reduce fuel consumption in light aircraft with modest speed reduction leading to reduced operating cost (increased miles per gallon), and to reduce cylinder head temperatures (CHTs) leading to improved engine longevity and reduced maintenance cost. A recent study by Chrisman (2011) using a limited number of data sets from aircraft in the Purdue University fleet of Cirrus SR20 training aircraft demonstrated statistically significant reduced fuel consumption and reduced CHT but no statistically definite speed reduction. This study confirms the earlier results for fuel consumption and CHTs using expanded data sets. It suggests that the failure to derive a statistically significant reduction in speed is a consequence of inherent experimental limitations rather than the lack of any reduction.
\end{abstract}

Keywords: lean of peak, LOP, rich of peak, ROP, best economy operation, general aviation, piston engine operation

\section{Introduction and Literature Review}

Best economy (BE, also known as lean of peak or LOP) operation of aircraft engines, rather than best power (BP, also known as rich of peak or ROP) operation, has been suggested as a means to reduce fuel consumption in light aircraft with modest speed reduction leading to reduced operating cost (increased miles per gallon), and to reduce cylinder head temperatures (CHTs) leading to improved engine longevity and reduced maintenance cost.

\footnotetext{
About the Authors

Ronald F. Brender is a current graduate student at Purdue University in Aviation and Aerospace Management. He has a BSE in Engineering Sciences, an MS in Applied Mathematics and a PhD in Computer and Communication Sciences, all from the University of Michigan. Dr. Brender also holds FAA CFI and CFII Certificates for Airplane Single Engine Land and was an adjunct faculty member in the School of Aviation Sciences at Daniel Webster College. Correspondence concerning this article should be sent to rbrender@purdue.edu.

Thomas Q. Carney is professor of Aviation Technology and former department head of the Department of Aviation Technology at Purdue University, where he has taught since 1972. He holds the Airline Transport Pilot certificate with multiengine, Mitsubishi Diamond, and Beechjet type ratings, and the Certified Flight Instructor certificate with airplane single- and multiengine, and instrument airplane ratings. In addition to his flight background, Dr. Carney holds MS and PhD degrees in Atmospheric Science, and maintains a courtesy faculty appointment in the Department of Earth and Atmospheric Sciences at Purdue.
} 
The physics and thermodynamics of spark ignition internal combustion air-cooled engines of the kind typically used in general aviation aircraft have been well-studied for many years and may be readily found in aviation maintenance textbooks and other sources (for example, Stone, 1993; Kroes \& Wild, 1995; Crane, 2005). The characteristics relevant to this study are shown in Figure 1 (Braly, 1999, Figure 1), which illustrates the relationships among exhaust gas temperature (EGT), cylinder head temperature (CHT), brake horsepower (BHP), and brake specific fuel consumption (BSFC) as a function of fuel flow for a single cylinder in normal operation, assuming manifold pressure and RPM are held constant. From Figure 1, it can be seen that best power fuel flow, which corresponds to the peak of the brake horsepower curve (labeled 16), occurs at a richer fuel flow than peak EGT (labeled 12). On the other hand, best economy, which corresponds to the minimum of the brake specific fuel consumption curve (labeled 18), occurs at a fuel flow leaner than peak EGT. The figure also shows that CHT will be lower at the best economy setting, compared to best

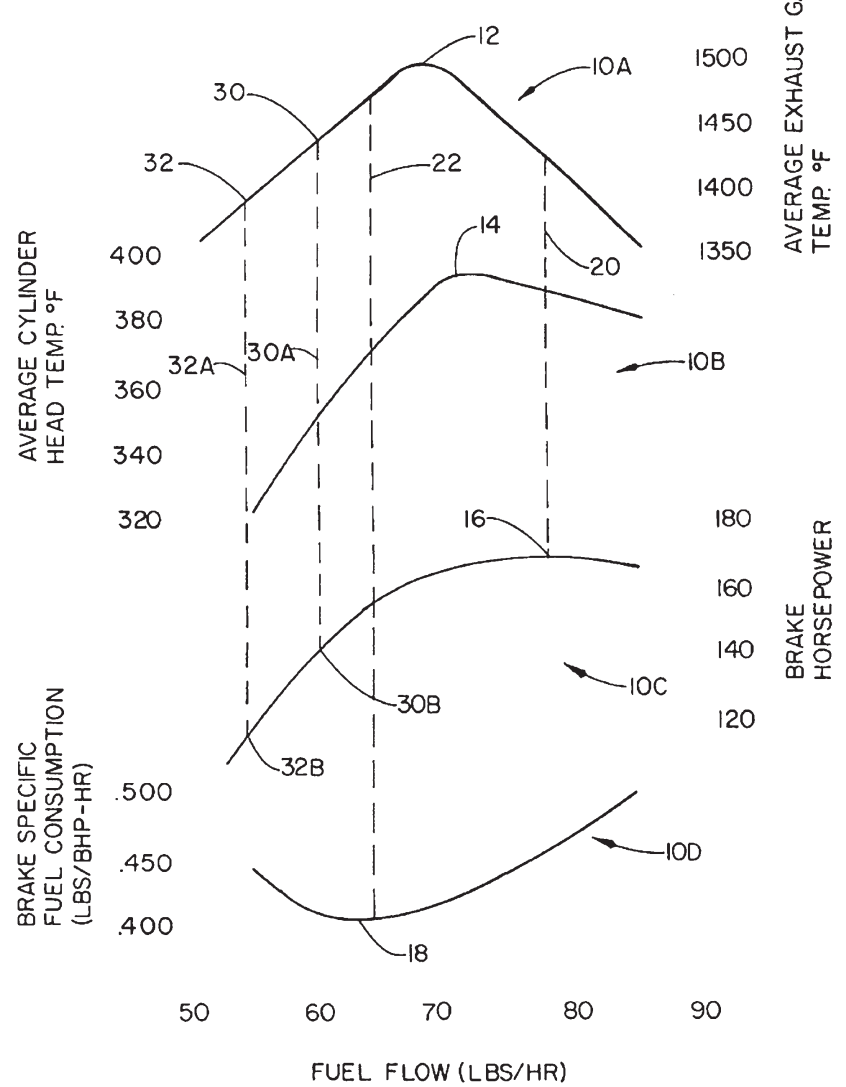

Figure 1. EGT, CHT, BHP and BSFC as a function of fuel flow ${ }^{1}$ (from Braly, 1999, Figure 1).

\footnotetext{
${ }^{1}$ The numeric values shown are for a specific aircraft engine, model IO550-B, as published by Teledyne Continental Motors. However, it is the shape and relationship of the several curves that matters here, and not the particular values.
}

power. Further, the figure shows that BHP will be lower at best economy setting, compared to best power, which may be expected to result in a lower true airspeed (TAS). Because the mass air flow involved in a combustion cycle will be constant for a given cylinder, manifold pressure, and RPM, the same relationships will be maintained and can be restated in terms of fuel-air ratio instead of fuel flow.

Routine operation using best economy settings in the piston general aviation fleet has long been problematic, often prevented by engine roughness and occasionally even detonation-related engine damage (Haines, 2010; Hirschman, 2009). Lycoming and Teledyne Continental, the two major piston engine manufacturers, as well as many engine overhaul shops, at one time considered lean of peak (LOP) operation potentially dangerous and grounds for voiding any engine warranty.

Inspired in part by work at the Wright Aeronautical Division of Curtiss Wright (1957) on the TC18 radial engine used on DC-7s, Braly (1999; n.d.) diagnosed most problems using best economy settings as the result of unequal fuel distribution among the several cylinders of an engine so that different cylinders are operating at different points along the curves of Figure 1 and thus producing unequal amounts of power. He explains, "On the rich side of peak EGT this imbalance is usually insignificant because the corresponding horsepower curve is typically flat in that range. On the lean side of peak EGT, however, the condition is usually significant because the corresponding horsepower curve typically drops off steeply in that range" (Braly, 1999). Braly developed and patented a technique for providing highly accurate, precisely-calibrated "balanced fuel injectors" that replace the original injectors to enable best economy operation. A digital engine monitor with EGT and CHT probes in each cylinder, as well as a digital fuel flow indicator (possibly combined), are also highly recommended, if not mandatory, for safe and efficient setting and monitoring of best economy operation.

Ratcliffe and Rogers (2002) measured engine vibration in a comparative inflight study using standard fuel injector nozzles and balanced flow fuel nozzles under typical cruise flight conditions in a Beechcraft Bonanza with a TCM IO550-BB engine. Their results indicate that balanced flow nozzles reduce engine vibration for most fuel/air ratios. This supports the notion that balanced nozzles help equalize power output among the multiple cylinders of an engine.

The literature suggests best economy is typically as much as $20 \%$ more fuel efficient compared to best power (Braly, n.d.; Hirschman, 2009; Horne \& Hirschman, 2011). Moreover, best economy mixtures result in lower cylinder head temperatures, which contribute to long-term engine health and reduced maintenance (Braly, n.d.; Hirschman, 2009; Curtiss-Wright Corporation, 1957). Hirschman (2009) suggests that LOP operation comes with a "cost" of about a 5\% reduction in airspeed and Braly (2012) suggests reductions as high as 8 to $10 \%$. 
Table 1

Summary of Chrisman's (2011) Key Results

\begin{tabular}{|c|c|c|c|c|}
\hline & Best Power (mean) & Best Economy (mean) & Reduction (mean) & Statistically Significant Difference (p $\square 0.05$ )? \\
\hline Fuel consumption $(\mathrm{GPH})$ & 10.933 & 8.792 & 2.141 & Yes \\
\hline Mean CHT $\left({ }^{\circ} \mathrm{F}\right)$ & 337.0 & 313.0 & 24.0 & Yes \\
\hline TAS (KT) & 133.09 & 131.83 & 1.26 & No \\
\hline
\end{tabular}

Purdue University operates a fleet of Cirrus SR20 aircraft, which are used for pilot training. The aircraft are all equipped with balanced injectors (Cirrus Design, 2008) and Garmin Cirrus Perspective Avionics (Garmin International, Inc., 2009) and thus are suitable for (and authorized to employ) lean of peak operation.

The SR20 Information Manual (Cirrus Design, 2008) describes the leaning procedure to establish best economy operation but does not provide the kind of detailed performance information (notably TAS and fuel flow to be expected for various combinations of density altitude, manifold pressure and engine RPM) provided for best power operation.

Chrisman (2011) studied the performance of SR20 aircraft used in the Purdue training fleet to confirm the ability to operate at best economy and to measure the (expected) reductions in fuel flow, CHT and TAS for best economy compared to best power operation. He arranged for student pilots and their instructors to use both LOP and ROP operation during the cruise portion of cross-country instructional flights. Further, he took advantage of the data logging capabilities of the Garmin Cirrus Perspective Avionics (Garmin International, Inc., 2009) to collect the needed aircraft flight performance and systems data.

Chrisman's main results are summarized in Table 1.

In short, Chrisman observed statistically significant (about $20 \%$ ) reduction in fuel flow and about $15^{\circ} \mathrm{F}$ reduction in cylinder head temperatures, both consistent with prior literature. He did not observe a statistically significant reduction in true airspeed, contrary to prior literature.

Limitations noted by Chrisman include the following:

- First, originally there were intended to be data from 64 flights (legs), half using best power and half using best economy settings during cruise. For various reasons, the final report made use of data from only 19 flights: nine using best economy and ten using best power. This is a smaller number of data sets than one might like.

- Second, because of the limited number of data sets, a "paired $t$ test [comparing best economy versus best power from the same round trip] was dropped in favor of a two-sample $t$ procedure" (p. 28).

- Third, the conclusion that there is no statistically significant difference in TAS when operating at best economy compared to best power is definitely at variance with prior literature. Chrisman correctly points out that the leaning procedure used may not match the theoretical model.

To understand this distinction it is useful to review the leaning process in detail. Consider again Figure 1. Imagine a vertical line that is initially placed near the right side of the figure. For the fuel flow indicated where the line intercepts the horizontal axis one can read the performance values that result. Now, slide the line slowly left-the gradually decreasing fuel flow is analogous to leaning the engine. As leaning progresses the following occur, in order:

- Initially, EGTs, CHTs and BHP all increase (power increases), while BSFC decreases (economy improves).

- BHP reaches a peak and starts to decrease. This peak is called best power (BP) and will result in the best airspeed.

- CHT reaches a peak and starts to decrease. This peak is of interest because higher CHT (which closely correlates with higher peak internal cylinder pressure) implies higher risk of detonation and greater stress on the engine. Peak CHT is generally the worst operating point for these reasons.

- EGT reaches a peak and starts to decrease. This peak corresponds to an ideal air to fuel ratio of 1:1 where there are exactly enough oxygen molecules to burn every fuel molecule, no more and no less. In reality, ideal combustion never occurs but the peak reflects the best that is achieved.

- BSFC reaches a minimum (economy peaks) and starts to decrease. This floor (peak) is called best economy and will result in the most distance travelled per unit of fuel consumed.

It is important to keep in mind that this figure assumes that engine RPM and MAP remain constant throughout the leaning process.

In practice, in an aircraft equipped with a constant speed propeller, the propeller governor will typically do a good job of maintaining constant RPM as leaning progresses. But, as BHP decreases there must inevitably be a reduction in airspeed, which in turn will result in a reduction in impact air pressure entering the engine, which will result in decreasing MAP. (This effect is mitigated in a turbocharged engine which tends to maintain a constant MAP as well as RPM, but the Cirrus SR20 engines are not turbocharged so this is not relevant here.) 
Historically, most aircraft with constant speed propellers have two separate pilot controls, the throttle for adjusting MAP and the propeller governor for adjusting RPM. After a pilot has leaned the mixture to achieve the best economy setting he will find that MAP has decreased. He can increase the throttle slightly to reestablish the original MAP, which will restore engine operation to the state described by the figure. And, because BHP is less at the best economy setting compared to best power (for the same RPM and MAP), airspeed will necessarily be less as well.

The Cirrus SR20 differs from most light general aviation aircraft in that it has a single power lever that controls both RPM and MAP (Cirrus, 2008).

The single-lever power control, labeled MAX-POWERIDLE, on the console adjusts the engine throttle setting in addition to automatically adjusting propeller speed. The lever is mechanically linked by cables to the air throttle body/fuel-metering valve and to the propeller governor. Moving the lever towards MAX opens the throttle butterfly and meters more fuel to the fuel manifold. A separate cable to the propeller governor adjusts the propeller oil pressure to increase engine RPM. The system is set to maintain approximately 2500 RPM throughout the cruise power settings and 2700 at full power. (p. 7-30)

The leaning procedure used in the Chrisman protocol is shown in the appendix of his study. These instructions were used to train instructors and were handed out as a supplementary checklist for inflight use. For best power, the procedure sets $65 \%$ power directly. For best economy, the procedure reads:

i. $\quad 70 \%$ power set at throttle

ii. Switch to engine page on MFD -1 . Engage lean assist function

iii. Lean at $1 \mathrm{gph} / \mathrm{sec}$

iv. Lean fuel/air mixture until "last peak"

v. Lean fuel/air to $50^{\circ} \mathrm{F}$ LOP

vi. If engine roughness occurs enrich fuel/air ratio until smooth operation resumes

vii. Verify engine power is at $65 \%$ *

$\cdots$

*Note that for best economy cruise settings are established, the power will typically decrease for $70 \%$ to $65 \%$. (Appendix, p. 49)

These steps clearly indicate the intent to end at $65 \%$ power. It anticipates the reduction in HP discussed above and deliberately starts "too high" in an effort to end up at $65 \%$ power. MAP, although available to the pilots (much less prominently displayed than Percent Power) is never considered at either the beginning or the end of the leaning process. Thus this procedure does not maintain constant MAP which is an assumption in the predictions for the performance impact of best economy. The result is to compare the TAS of two $65 \%$ power settings, one best power and one best economy, from which the lack of a TAS difference should not be a surprise.

Because of these issues, Chrisman did not conduct the experiment that he thought he was conducting. But he did conduct a useful experiment. Because percent power is the primary engine performance indication presented to a Cirrus SR20 pilot, leaning by reference to that number is the "natural" way to manage that type of airplane/power plant. Thus the experiment does usefully relate to the way the Cirrus aircraft are typically operated.

The actual power setting cannot be measured directly by the airplane sensors but is derived using an algorithm employing manifold pressure, indicated airspeed, outside air temperature, pressure altitude, engine speed, and fuel flow (Cirrus Design, 2008). The actual algorithm is not published, and therefore not subject to review ${ }^{2}$. Finally, the leaning procedure is a manual process carried out by a pilot in the midst of a flight and is therefore subject to variations in pilot technique and environmental distractions.

It is a goal of this study to use an engine performance model to compute adjusted values that are more suitable for testing the theory in Figure 1. The hypothesis is that this will explain why Chrisman failed to observe a significant difference in TAS.

Finally, Chrisman considered all flights equally without regard to which aircraft was being flown. This admits the possibility that a single aircraft used for a disproportionate number of flights might skew the results in a manner not representative of the fleet as a whole.

It is a goal of the current study to perform a modified analysis in which data for each specific aircraft are first derived and the results combined with equal weight relative to other aircraft. There is a possibility that this analysis might identify a particular aircraft that is sufficiently different from the others to warrant maintenance evaluation.

\section{Research Questions}

This research focuses on three research questions. For a given combination of manifold pressure (MAP) and engine speed (RPM):

Is reduced fuel consumption achieved by operating at best economy during the cruise portion of flight, compared to operating at best power?

Are cylinder head temperatures lower during best economy settings, compared to best power operation?

\footnotetext{
${ }^{2}$ A request to Cirrus Aircraft for details of the calculation was denied on the grounds that the information was considered proprietary.
} 
Is there a loss in true airspeed during best economy settings, compared to true airspeed during best power output?

These questions are nearly identical to those in the predecessor study by Chrisman (2011) with one key difference: this study seeks to compare best economy versus best power for the same combination of MAP and RPM while Chrisman compared best economy versus best power for the same power setting.

\section{Methodology}

\section{Data Collection}

This study includes experimental flights beyond those originally conducted as part of the Chrisman protocol. Because very limited records are available regarding the flights intended for use but not actually used in his study, the task of data collection initially took the character of data re-identification. It later became much broader.

The initial concept was to collect data sets for all flights by the SR20 aircraft (based on their aircraft ID, or Nnumber) that took place between any of the airports authorized in the Purdue University Flight Operations Handbook (Department of Aviation Technology, 2011, p. 5-5) for conducting student solo cross country flights. History logs from www.flightaware.com covering the

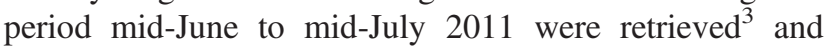
used to identify candidate flights based on origin, destination and aircraft ID. For simplicity, all of the data sets for a date of interest were collected from each respective aircraft, rather than just the smaller set of specific flights.

The collection of additional flight data sets proved fortuitous as it became clear that the data analysis methodology was almost fully automated and not laborintensive. Many additional flights proved interesting and of use, well beyond those in Chrisman's protocol.

The data collected here may be regarded as a convenience sample with a bias toward including flights thought likely to include use of best economy operation. Because the relative numbers of $\mathrm{BP}$ versus $\mathrm{BE}$ operations is not of interest of itself, the bias serves solely to promote finding a sufficient large number of BE flights to support statistically significant results.

\section{Data Analysis}

Data analysis was performed using a series of software programs written specifically for this study. All but the last of these programs were written in the Java programming language using the Eclipse Integrated Development

\footnotetext{
${ }^{3}$ www.flightaware.com history logs are limited to three months of prior history, so history for that period is no longer available online. Printouts were made and retained, however.
}

Environment (Eclipse Foundation, 2012). The last was written in the $\mathrm{R}$ language and environment ( $\mathrm{R}$ Development Core Team, 2012) to perform the final statistical analysis steps, including preparation of most of the figures used in this report.

This section has three parts. The first describes the key issues addressed and methodologies adopted in order to completely automate the analysis. The second describes how the software was organized to accomplish the analysis. The third describes the statistical analysis that was carried out by the last component of the software.

\section{Key Issues}

\section{Data File Naming and Management}

Cirrus Perspective SR20 aircraft equipped with Perspective Avionics create a new data file each time aircraft power is turned on. The file name is formed as illustrated in this example: log_090210_104506_KLAF.csv. The first group of numbers specify the local date (yymmdd; February 10, 2009 in this example) and the second group of numbers specify the local 24-hour time (hhmmss; 10:45:06AM in this example $)^{4}$. The final letters specify the airport identifier where the airplane is located, if known, or blank otherwise. The file type csv indicates that the file uses the "comma separated value" formatting conventions, which are commonly readable by a variety of spreadsheet and other types of applications.

Examination of these data sets revealed that the first line of data includes key identification and version number information. The very first cell appears intended to identify the applicable aircraft but does not. This means that combining data files from multiple aircraft in a single computer directory loses track of the applicable aircraft.

\section{Stable Regions}

Figure 2 shows a typical display of engine operating parameters during a complete flight. This was a roundrobin flight between Lafayette, IN (KLAF) and Champaign-Urbana, IL (KCMI).

Chrisman discusses in detail the need for "conditioning" the data to find a suitable point at which FF, CHTs and TAS values can be extracted for use in the subsequent analysis. He illustrates how key parameters change and can be used to identify the successive transitions from climb, level off at altitude, through cruise mixture adjustment and stabilization of CHTs. This process required visual analysis of graphs such as shown in Figure 2, so was somewhat subjective and was highly labor-intensive.

\footnotetext{
${ }^{4}$ Data logging starts as soon as power is turned on but the GPS takes time (generally a minute or so) to obtain an initial position fix (which includes a precise date and time); the date and time used in the filename corresponds to when that fix is first available. The airport identifier may not be determined until some minutes later.
} 


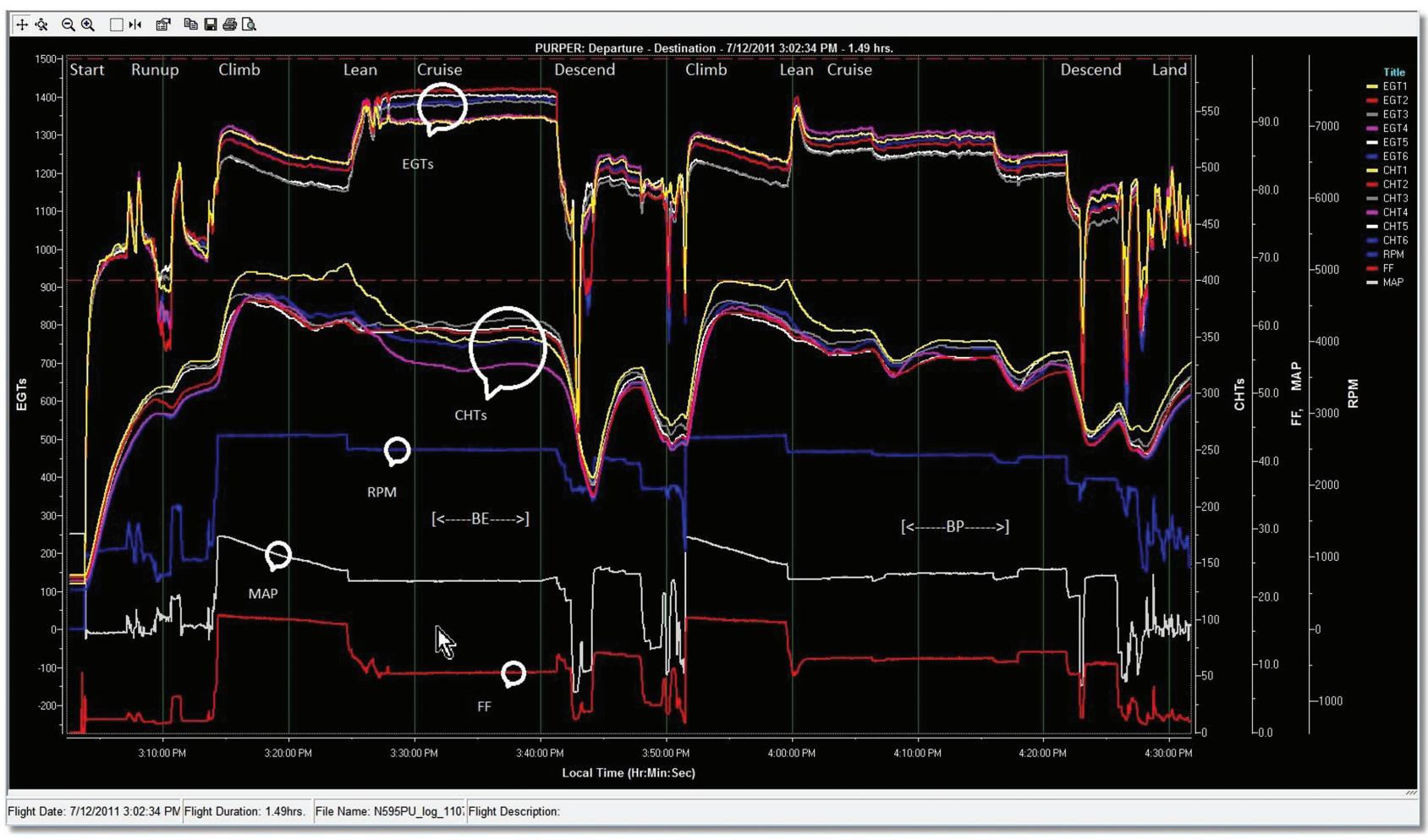

Figure 2. Typical display of engine data set.

In this study, an alternative approach was taken to automatically identify stable regions suitable for subsequent statistical analysis. A stable region is defined as a period of at least five minutes during a flight, in which:

1. Altitude is constant, plus or minus 50 feet

2. Fuel flow is constant, plus or minus .25 gallons per hour $(\mathrm{GPH})$

3. Engine speed is constant, plus or minus 25 RPM

4. Manifold pressure (MAP) is constant, plus or minus .25 inches of $\mathrm{Hg}$

5. Average cylinder head temperatures (CHTs) are constant, plus or minus 5 degrees Fahrenheit

The values specified here are chosen to be roughly two to three times the measurement and/or control accuracy of the respective parameters, so that normal measurement variability and jitter would not preclude finding any stable regions. These specific values are somewhat subjective based on the researcher's personal experience using similar engine monitoring equipment and not on a detailed study.

\section{Identification of BP versus BE Regions}

Chrisman identified best power versus best economy usage in two different ways. Initially, he specifically selected (randomly) which was to be used on each leg. However, he found that on numerous occasions the pilots failed to follow that selection and selected for themselves.
Alternatively, based on early results he concluded that fuel flow and CHTs were in fact clearly lower during best economy segments so he resorted to using them to differentiate (consistent with the literature).

This study follows the precedent set by Chrisman except that the only criterion used is fuel flow. The Cirrus SR20 Information Manual (Cirrus Design, 2008) provides tables for Cruise Performance (pp. 5-21, 5-22 and 5-24) and Range/Endurance Profile that show $65 \%$ best power operations always consume at least $10.4 \mathrm{GPH}$. No best economy Cruise Performance tables are given, but one best economy Range/Endurance Profile table (p. 5-24), shows all fuel flows at 8.4 GPH. This study classifies fuel flows of at least 9.5 gallons per hour (GPH) as Best Power and fuel flows of less than 9.5 GPH as Best Economy. Additional justification for this choice, based on the data collected, is given in the discussion following. Alternative attempts to classify stable regions by means other than fuel flow are also discussed.

\section{Software Organization and Operation}

Software developed for this study consisted of four parts or steps. These are discussed in turn.

The Step 1 program reads the original data set and rewrites it to insert the aircraft ID into the first field of the data set, and to add the aircraft ID as a prefix to the file name. 
The Step 2 program scans the GPS LAT/LON information in the data set to determine at what airports the aircraft landed during the flight. The sequence of airport identifiers is appended to the file name. An aircraft is considered to have landed if the aircraft is within 1 second (approximately 1 mile) of the airport location (latitude and longitude) and aircraft airspeed is less than 40 knots. Aircraft altitude compared to the airport elevation could have been used as part of the criteria but proved unnecessary in practice.

This step provided a more intuitively useful name than just date and time numbering. In addition, the airport information was used to filter out and discard flights that did not involve more than one airport.

The Step 3 program performs the heart of the data analysis process.

The data set is read and summary information is collected for each six-second region or zone of the file. Zones were introduced as a way to limit the main memory needed for processing. Six seconds was chosen as long enough to be able to detect local trends but not so long as to contaminate large time segments because of local effects 5 .

Each zone includes the following information:

- Minimum and maximum altitude

- Minimum and maximum engine RPM

- Minimum and maximum fuel flow

- Minimum and maximum manifold pressure

- Minimum and maximum average CHTs

- Minimum and maximum true airspeed

- Minimum and maximum outside air temperature (OAT)

- Average EGTs at the beginning and end of the zone

- Fuel flow at the beginning and end of the zone

Each zone is tested for being unstable (not satisfying the criteria region criteria above) by itself. Unstable zones are excluded from subsequent analysis.

Other zones are assigned a heuristic figure of merit computed as the sum of the variability of each of the five parameters used to determine stability. The variability of a parameter is the difference between the maximum and minimum values (always positive) divided by the average of the maximum and minimum. It can be seen that if all five parameters are perfectly constant then the total variability will be zero. Further, if each of the parameters has a maximum and minimum that just satisfies the worst case stability criterion, the total variability will be at a maximum.

Zones are then considered in order from best to worst variability. Each zone is treated as a "seed" for "growing" a stable region. First, a scan forward and backward attempts to confirm that there are enough adjacent zones not already

\footnotetext{
${ }^{5}$ Zone durations of 2, 4, 6, 10, 15 and 30 seconds were tried before settling on a size of 6 .
}

unstable and not already used in a previously found stable region. If not, all of the scanned zones are marked as unavailable to preclude further consideration (a waste of effort).

If a large enough stable region is possible, then a second scan attempts to "grow" the region first forward and then backward from the initial seed. In each case, a new zone is combined with the growing candidate region only if the data values across that whole region continue to qualify as a stable region.

When the largest possible candidate region has been determined, it is checked for being at least five minutes in length. If so, a stable region has been successfully formed. All of the zones included in the new region become unavailable for consideration in any other stable region. If the region is too short, then its zones remain available for reconsideration starting with some later seed in the hope that either the beginning or ending part of it may be included.

The last part of this step is to output a file (a csv file that is readable as an Excel spreadsheet) that summarizes all of the stable regions identified together with all of the associated properties needed for performing statistical analysis.

The Step 4 program (an R script) performs the following statistical analysis.

\section{Fuel Consumption and CHTs}

A two-sample $t$ test was used to compare fuel consumption and average CHTs from best economy and best power operation. For the more limited data sets in which both best economy and best power stable regions are found in the same flight, a paired $t$ test was used.

\section{True Airspeed Analysis}

Starting with the recorded value of manifold pressure during intended best economy operation, a model of engine performance was sought to calculate what engine power would result from adjusting MAP to match that used during the corresponding best power flight. It was hoped this model could be based on the algorithm used in the Garmin avionics to calculate percent power (and validated based on known engine design principles). Otherwise, a model would be derived, if possible, from engine design principles directly.

\section{Per Aircraft Aggregation Analysis}

The same analysis was repeated except that data for each airplane was separately aggregated and compared with other aircraft.

\section{Results}

Starting with 247 raw data sets, 175 were eliminated (by Step 2 above) because they did not involve a flight between 
two (or more) airports ${ }^{6}$. Of the remaining 72 data sets, Step 3 automated analysis found a total of 55 data sets that contained one or more stable regions. Of those stable regions, 104 were classified as best power operations and 23 as best economy operations.

\section{Fuel Flow Statistics}

Fuel consumption rates for best economy and best power are presented below along with descriptive statistics.

Figure 3 combines three histograms on a common scale: Data for best economy are shaded using /, data for best power operations using $\backslash$ and the total of both using the overall outline. The overall outline shows that there definitely appear to be two distinct subpopulations. Detailed examination of the data shows that there are no values between 9.25 and $9.75 \mathrm{GPH}$, which supports the use of $9.5 \mathrm{GPH}$ as a dividing threshold.

Two-sample $t$ test statistics indicate that the mean best economy fuel flow of $8.69 \mathrm{GPH}$ is less than the mean best power fuel flow of 10.97 GPH $(\mathrm{t}=-28.88$, df $=118.771$, $\mathrm{p}<2.2 \mathrm{e}-16)$.

\section{Cylinder Head Temperatures}

Average cylinder head temperatures for best economy and best power are presented below along with descriptive statistics.

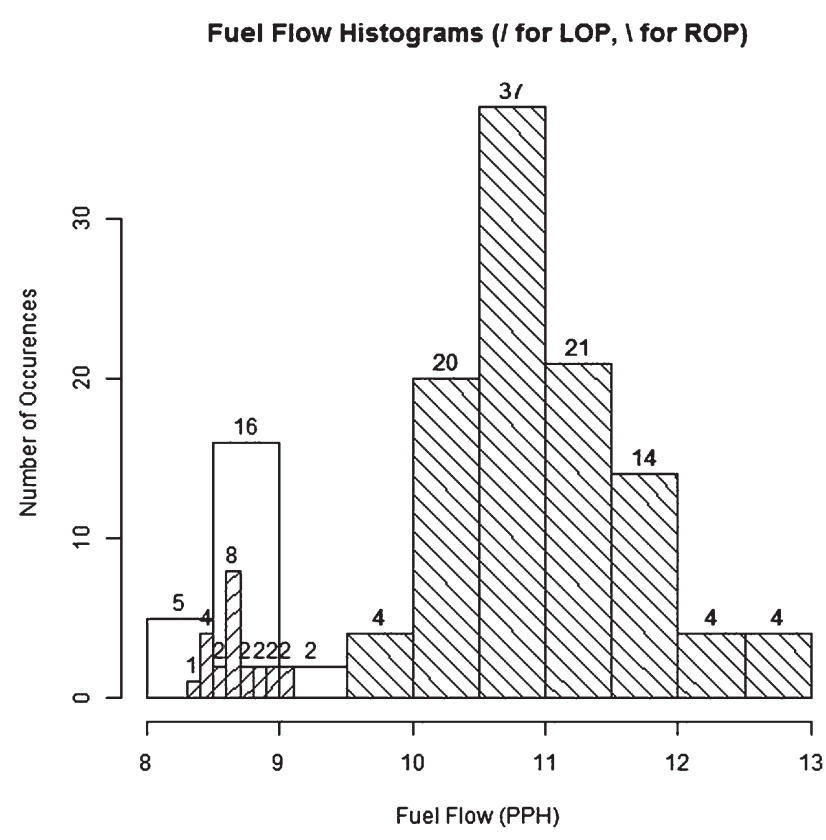

Figure 3. Fuel flow histograms.

\footnotetext{
${ }^{6}$ These data sets are taken from a training fleet in which a high percentage of flights remain close to the Lafayette departure airport, especially for those training for Private and Commercial certificates. It is assumed that flights not between airports are highly unlikely to include a long enough period of steady-state flight to be useful during subsequent analysis.
}

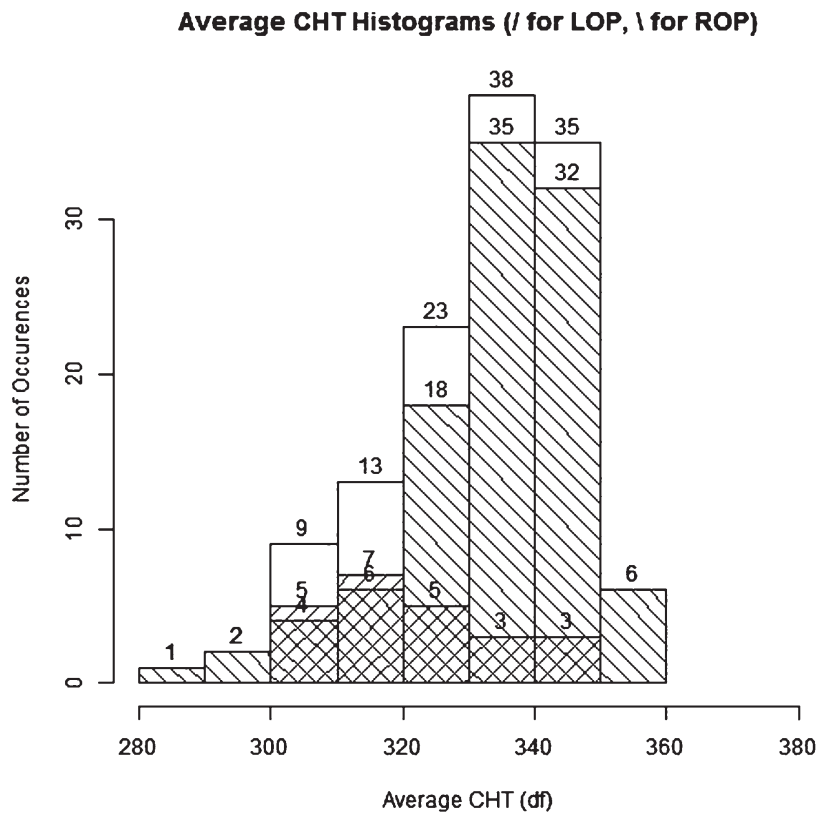

Figure 4. Average cylinder head temperatures.

Figure 4 combines three histograms on a common scale: Data for best economy are shaded using /, data for best power operations using $\backslash$ and the total of both using the overall outline.

Two-sample $t$ test statistics indicate that the best economy average cylinder head temperature of $321.6^{\circ} \mathrm{F}$ is less than the best power average cylinder head temperature of $334.5^{\circ} \mathrm{F}(\mathrm{t}=-4.0255$, df $=323.6, \mathrm{p}=0.00031)$.

\section{True Airspeed}

True airspeed best economy and best power are presented below along with descriptive statistics.

Figure 5 combines three histograms on a common scale: Data for best economy are shaded using /, data for best power operations using $\backslash$ and the total of both using the overall outline.

Two-sample $t$ test statistics $d o$ indicate that the means for best economy and best power TAS are different $(\mathrm{t}=$ -2.8484 , df $=41.467, \mathrm{p}=0.00681$ ).

However, despite this statistic, the histogram indicates such a broad distribution of air speeds, including some ROP airspeeds that are less than the lowest LOP airspeeds, that these simple statistics are not telling the whole story.

\section{Per Aircraft Aggregation}

The training fleet includes 16 SR20 aircraft with identification numbers from N580PU through N595PU. As seen in Table 2, only four aircraft, (N584PU, N593PU, N594PU, and N595PU) have enough samples to allow tentative comparison on an aircraft-by-aircraft basis.

Matched pairs $t$ tests among the four groups for both LOP and ROP regions showed few statistically significant differ- 


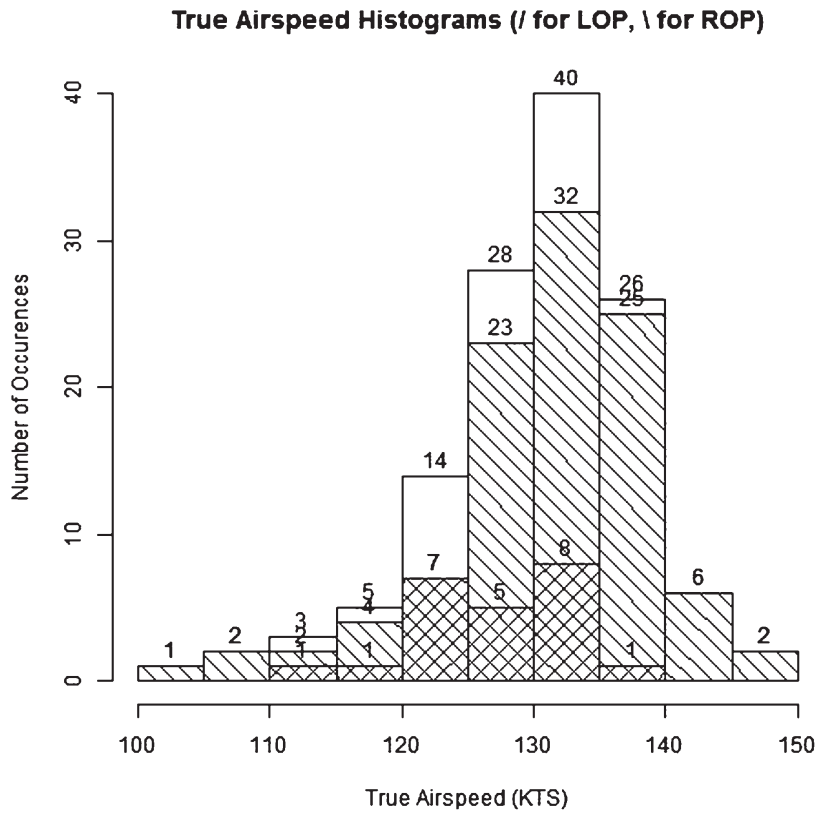

Figure 5. True airspeed histograms.

ences. Even where the differences are statistically significant, they remain well within the overall ranges established above.

\section{Comparison With Chrisman Results}

The results of the previous sections are summarized in Table 3. Comparison with the results from Chrisman shown in Table 1 indicates substantially similar results, except that a statistically significant difference in TAS is found $(\mathrm{t}=-2.8484, \mathrm{df}=41.467, \mathrm{p}=0.00681)$. While this appears to contradict the results in Chrisman, this conclusion is not clear as will be seen below.

\section{Matched Pairs Analysis}

The automated results include nine flights in which both a LOP and ROP stable region were identified. This subset of the data is especially interesting because it should reduce much of the variability (such as different aircraft, pilot technique, environmental conditions, and so on) that is not controlled in the earlier analysis. The results of matched pairs $t$ test comparisons are summarized in Table 4 .

Note that the TAS reduction of 3.17 knots is smaller than indicated in the larger sample and, more importantly, fails to meet the test for being statistically significant (though not by much). See the later discussion for further analysis.

\section{Discussion}

\section{The Truth About True Airspeed}

The measurement and usage of comparable airspeed information across aircraft and across flights is subject to numerous confounding factors. On the favorable side, all of the aircraft involved are of the same make and model. Further, they were all purchased and placed in common service at the same time and at the same location. Thus variations between aircraft related to manufacturing and operational history are assumed to be negligible.

It may be useful to note that indicated airspeed is recorded in the data sets to a precision of 0.01 knots while true airspeed is recorded to a precision of 1 knot.

\section{True Airspeed Versus Horsepower}

From basic aerodynamics it is known (other things being equal) that in the normal cruise airspeed range the largest

Table 2

Stable Region Count by Aircraft

\begin{tabular}{lcccccccrr}
\hline Aircraft & N583PU & N584PU & N586PU & N587PU & N588PU & N591PU & N593PU & N594PU & N595PU \\
\hline LOP Stable Regions & 0 & 8 & 2 & 1 & 0 & 0 & 4 & 4 & 4 \\
ROP Stable Regions & 5 & 5 & 0 & 8 & 3 & 1 & 24 & 24 & 38 \\
\hline
\end{tabular}

Table 3

Summary of Key Results

\begin{tabular}{lcccc}
\hline & Best Power (mean) & Best Economy (mean) & Reduction (mean) & $\begin{array}{c}\text { Statistically Significant Difference } \\
(\mathrm{p}<0.05) ?\end{array}$ \\
\hline Fuel consumption $(\mathrm{GPH})$ & 10.97 & 8.69 & 2.28 & Yes \\
Maximum CHT $\left({ }^{\circ} \mathrm{F}\right)$ & 334.5 & 321.6 & 12.9 & Yes \\
TAS $(\mathrm{KT})$ & 131.6 & 127.5 & 4.1 & Yes \\
\hline
\end{tabular}

Table 4

Matched Pairs Comparisons Key Results

\begin{tabular}{lcc}
\hline & Reduction (mean) & Statistically Significant Difference $(\mathrm{p}<0.05) ?$ \\
\hline Fuel consumption $(\mathrm{GPH})$ & 1.988 & Yes \\
Maximum CHT $\left({ }^{\circ} \mathrm{F}\right)$ & 18.83 & Yes \\
TAS $(\mathrm{KT})$ & 3.17 & No $(\mathrm{p}=0.08211)$ \\
\hline
\end{tabular}


component of drag is parasite drag, which is proportional to the square of velocity. Because power required is proportional to drag multiplied by velocity, it follows that power required is proportional to the cube of velocity (Smith, 1992).

$$
\begin{gathered}
\mathrm{D} \propto \mathrm{V}^{2} \\
\mathrm{P}=\mathrm{D} * \mathrm{~V} \propto \mathrm{V}^{3}
\end{gathered}
$$

It follows that it takes a relatively large change in power to make a small change in airspeed. To illustrate, a $5 \%$ decrease (a factor of .95) in airspeed (such as is predicted by prior literature) corresponds to almost a $14 \%$ decrease in power $\left(0.95^{3}=0.857\right)$ such as from $65 \%$ power to $56 \%$ power $\left(0.65^{*} 0.857\right)^{7}$. Using the Brake Horsepower scale in Figure 1, we see a reduction from about $170 \mathrm{HP}$ at best power (labeled 16) to about $140 \mathrm{HP}$ at best economy (labeled 30B), an 18\% reduction.

Measuring small changes in airspeed is confounded by many factors. Further, horsepower is not directly measured in light general aviation aircraft and must be computed from several other factors, notably engine RPM and MAP as well as temperature, which themselves are subject to instrumentation errors.

One conclusion is that reliable measurements related to the kind of small changes in airspeed observed and of interest in this study are unlikely unless very carefully conducted.

It is a limitation of this study (as well as Chrisman's) that engine power is not available in the output data sets. Given that Chrisman's protocol depended strongly on pilot use of the computed horsepower (as a percent of maximum) displayed by the Garmin avionics it is most unfortunate that the same information is not also recorded for later analysis.

Two models were developed in an unsuccessful attempt to compute engine power and its associated TAS and fuel flow. Finding or developing a viable horsepower model proved beyond the resources of this study.

\section{POH Model}

The Cruise Performance table in the SR20 Information Manual (Cirrus Design, 2008, pp. 5-21 and 5-22) can be regarded as a kind of performance model for best power (ROP only) operation-given pressure altitude, OAT, MAP and RPM, one can look up the resulting expected percent horsepower, true airspeed and fuel flow. A software implementation, including multidimensional interpolation, was created and validated to produce outputs that closely match the $\mathrm{POH}$ table values.

\footnotetext{
${ }^{7}$ Bear in mind that engine power output in the normal cruise operating range for light aircraft piston engines is rarely less than $50 \%$ nor more than $80 \%$--an increment or decrement of $9 \%$, nearly a third of this range, is a major change.
}

Attempts to correlate the predicted true airspeed and fuel flow against values measured inflight as an indirect means of validating the horsepower calculation showed poor accuracy and major scatter. The discrepancies were never resolved.

Even if the POH model had worked as hoped, it does not address best economy (LOP) operation.

\section{Perry Model}

A Cirrus engineer provided references (personal communication by way of Professor Michael Suckow, 2011) for a non-proprietary engine performance model developed by James Perry (Sequoia Aircraft Corp, 1990) together with Cirrus-specific model parameters ${ }^{8}$ for the TCM IO-360-ES engine used in the SR20 aircraft. A software implementation of this model, augmented with a small "fudge factor" (less than 3\%), showed an extremely close match to the $\mathrm{POH}$ model described in the previous section.

While this provides mutual validation with the $\mathrm{POH}$ model, it leaves unexplained the $\mathrm{POH}$ model discrepancies. And, of course, the Perry model also does not address best economy (LOP) operation.

\section{True Airspeed Versus Weight}

From basic aerodynamics, it is also known that (other things being equal), the ratio of two airspeeds is proportional to the square root of the ratio of the two corresponding weights (Smith, 1992).

$$
\mathrm{V}_{1} / \mathrm{V}_{2} \propto \sqrt{ }\left(\mathrm{W}_{1} / \mathrm{W}_{2}\right)
$$

We can roughly estimate the typical change in weight during a simple out and back cross country flight. Assume a one hour leg in each direction and an average fuel burn of 10 gals per hour (and ignore extra fuel for climb). The SR20 basic empty weight is approximately $2050 \mathrm{lbs}$ (Cirrus Design, 2008). Add $340 \mathrm{lbs}$ for two (FAA standard) people and $336 \mathrm{lbs}$ for full fuel (required by Purdue University's flight policies) to get a takeoff weight of about $2730 \mathrm{lbs}$. We estimate a mid-flight weight of 2730-30 or $2700 \mathrm{lbs}$ for the outbound leg and 2730-90 or $2640 \mathrm{lbs}$ for the return leg. The square root of the weight ratio, .989 , suggests that a further source of air speed measurement error on the order of $1 \%$ can result simply from the fuel burn that takes place during a cross country flight.

In the data sets used here (and by Chrisman), weight is neither measured nor otherwise available. Further, even in the matched pairs analysis presented here, the order of the LOP versus ROP stable regions was not considered. Fortunately this effect is small, and in light of other confounding factors, can be neglected.

\footnotetext{
${ }^{8}$ Only the frictional horsepower coefficient was not otherwise available from the TCM Model IO-360 Series Maintenance and Operator's Manual (1994).
} 


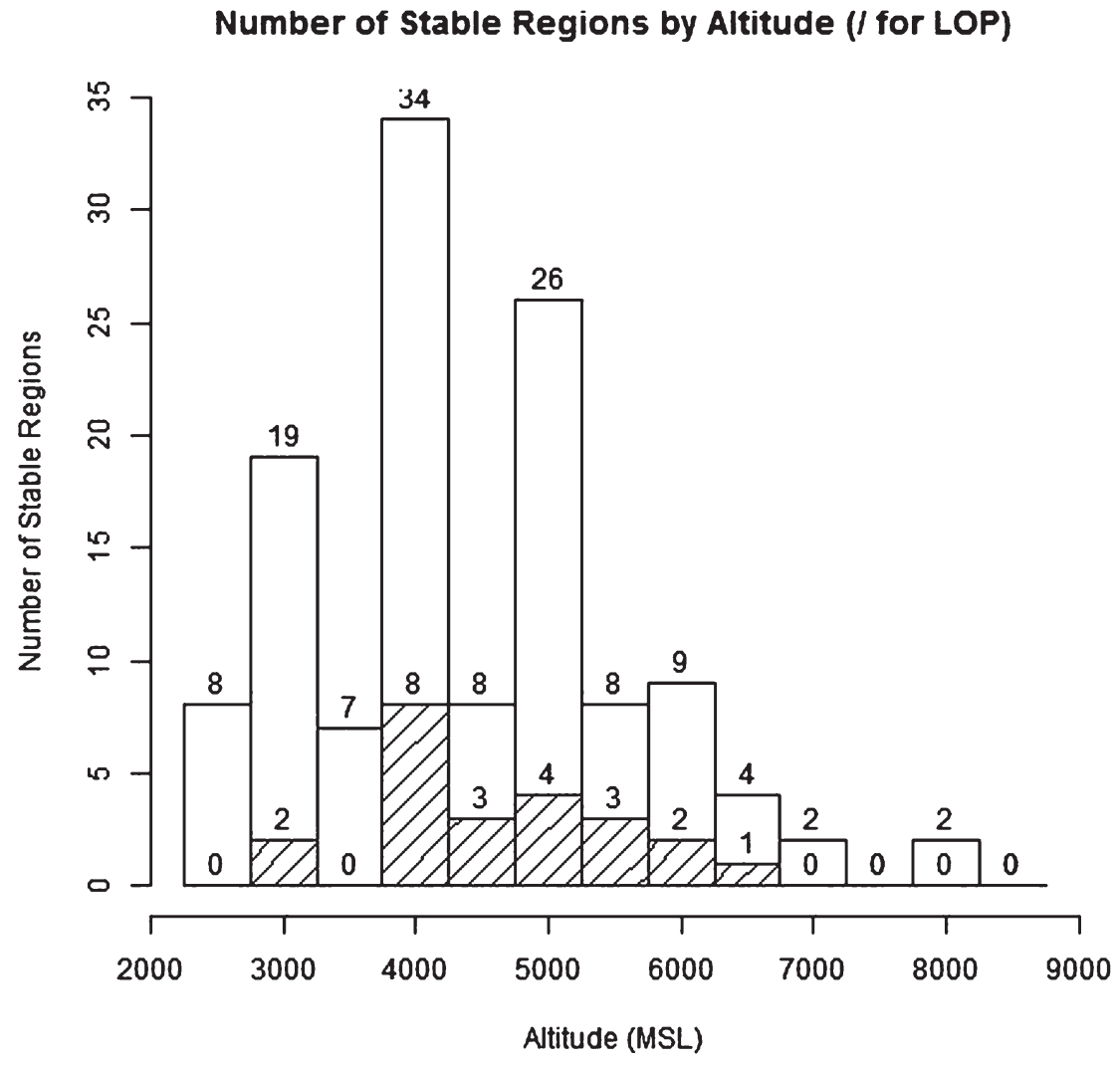

Figure 6 . Stable regions by altitude.

\section{True Airspeed Versus Altitude}

From basic aerodynamics it is known (other things being equal) that true airspeed varies with air density, which depends primarily on altitude (pressure), but also temperature and humidity (Smith, 1992). A common rule of thumb is that for a constant indicated airspeed, true airspeed increases by $2 \%$ per 1000 feet of altitude.

The flights in this study varied from 2500' MSL to 8000' MSL. The flights in Chrisman's study varied from 3000' MSL to 6000' MSL. This suggests that variability of as much as $11 \%$ (6\% in the Chrisman data) might be confounding the analysis, which is definitely much larger than the measured mean effect.

To explore this possibility, consider Figure 6, which shows the distribution of stable regions by altitude, at 500' intervals. Two altitudes, 4000' MSL and possibly 5000' MSL, appear to have enough samples to provide additional insight, but the others have too few data points to be statistically interesting.

Table 5 and Table 6 summarize the key results from $t$ tests applied to the subsets of data for 4000' MSL and 5000' MSL, respectively. In both cases, the differences for TAS are statistically significant $(\mathrm{t}=-3.0418$, df $=$ $11.398, \mathrm{p}=01081)$ and $(\mathrm{t}=-2.9006, \mathrm{df}=23.089, \mathrm{p}=$ $0.00804)$, respectively. This supports the suggestion that altitude is a significant confounding factor in the analysis.

\section{A Perspective on Common Data}

The work described here was motivated and inspired by Chrisman, but little that has gone before has considered how his data sets relate to these results. Working notes provided by Chrisman (personal communication) were sufficient to identify the original data set from which each of his data points was taken. Of Chrisman's 19 data samples:

Table 5

Summary of Key Results at 4000' MSL

\begin{tabular}{|c|c|c|c|c|}
\hline 4000’'MSL & Best Power (mean) & Best Economy (mean) & Reduction (mean) & $\begin{array}{l}\text { Statistically Significant Difference } \\
\qquad(\mathrm{p}<0.05) ?\end{array}$ \\
\hline Fuel consumption $(\mathrm{GPH})$ & 10.86 & 8.59 & 2.27 & Yes \\
\hline Maximum CHT $\left({ }^{\circ} \mathrm{F}\right)$ & 335.4 & 314.8 & 20.6 & Yes \\
\hline TAS (KT) & 132.3 & 125.4 & 6.9 & Yes \\
\hline
\end{tabular}


Table 6

Summary of Key Results at 5000' MSL

\begin{tabular}{|c|c|c|c|c|}
\hline 5000' MSL & Best Power (mean) & Best Economy (mean) & Reduction (mean) & Statistically Significant Difference $(\mathrm{p}<0.05)$ ? \\
\hline Fuel consumption (GPH) & 10.82 & 8.76 & 2.06 & Yes \\
\hline Maximum $\mathrm{CHT}\left({ }^{\circ} \mathrm{F}\right)$ & 341.9 & 316.0 & 25.9 & Yes \\
\hline TAS (KT) & 134.2 & 131.3 & 2.9 & Yes \\
\hline
\end{tabular}

- Eight were "accepted" and used here.

- Four were not accepted because there was not a long enough stable period surrounding that point to qualify as a stable region as defined here.

- Five were not accepted because the cylinder head temperatures (CHTs) were too variable ${ }^{9}$.

- One was accidently omitted during initial data collection.

\section{Further Research}

There are several future directions to pursue.

\section{Classification of LOP Regions}

For the purposes of this study, the classification of a stable region as using best economy versus best power operation is based on a simple threshold level of fuel flow. This is not unreasonable because it is known in advance that all of the aircraft involved are nearly identical (that is, they are all the same type of aircraft with the same type of engine). For more general application it would be attractive if this classification could be made using only data available in the data log itself.

One approach is try to investigate the pattern of EGT behavior (increasing to a peak and then decreasing) while fuel flow declines in the period just prior to the beginning of a stable region. Several attempts to accomplish this were coded, with limited success. On the positive side, one algorithm was successful for seven of the 23 LOP stable regions.

Examination of regions where this algorithm failed suggested several kinds of possible improvement. First, some stable regions appear to be continuations of an earlier stable region. That is, a prior stable region ends because of some transient disturbance of the stability criteria (perhaps a turn or quickly corrected altitude deviation) after which the aircraft soon settles back into its earlier stable state. Such stable regions might be merged using some kind of more relaxed stability criteria. (This has the perverse consequence of raising the success rate for recognition of the leaning pattern at the expense of reducing the number of LOP stable regions. But then such merging would apply to ROP regions as well.)

\footnotetext{
${ }^{9}$ In four of these cases, the CHT instability appears to be synchronized with wandering TAS and altitude. One might speculate that this resulted from hand flying rather than use of the autopilot-most of the flights studied here are student training flights, after all, but there is no way to know who, or what, was at the controls at any given time.
}

Second, the skill and rate at which a pilot performing the leaning process can be quite variable, sometimes overshooting and restarting, sometimes being interrupted by other demands. This suggests that the pattern to be recognized may be hard to characterize and hard to recognize without resorting to much more sophisticated pattern recognition software technology.

Third, and perhaps most profoundly, some LOP stable regions evidence no discernible leaning behavior. This can result when an experienced pilot flying a familiar airplane establishes LOP operation in but a second or two based on prior experience. In this case, the once-per-second sampling rate common to most aircraft data logging systems would mostly or totally miss the intermediate transitions.

\section{Bigger Data Needs Horsepower Models}

It is always easy to suggest applying research to a larger set of data samples. In this case, that is quite easy to do because more than two years of data are now available from Purdue University's training fleet and software tools make analysis relatively easy. However, further investigation of LOP versus ROP operation will not really be valuable until better information on engine power output can be obtained.

\section{Broader, not Just Deeper, Horizons}

This research has demonstrated that interesting analysis concerning general aviation aircraft operation can be automatically carried out based on information available in data sets now routinely created by many glass panel equipped aircraft. Examination of the data sets created by the Cirrus Perspective by Garmin Cockpit avionics shows that the available data are not limited to just engine operation or even purely engineering information. Information on avionics setup (such as communication and navigation frequencies selected) and course flown may be useful for studying a variety of human factors issues, including evaluation of pilot performance and perhaps, someday, even pilot aeronautical decision making.

\section{Conclusions}

This study confirmed, using a much larger set of data, two key results of Chrisman's prior study: best economy or LOP engine operation does reduce fuel flow compared to best power or ROP operation and does reduce engine cylinder head temperatures. It also shows that LOP operation appears to be associated with a small reduction 
in TAS compared to ROP operation, but this is ambiguous because of multiple confounding effects that impact TAS and the lack of good HP information either in, or derivable from, the recorded data. These results were obtained using completely automated techniques that offer the possibility of analyzing much larger data sets.

\section{References}

Braly, G. W. (1999). U.S. Patent No. 5,941,222. Washington, DC: U.S. Patent and Trademark Office.

Braly, G. W. (2012). U.S. Patent Application No. 13/528,113. Washington, DC: U.S. Patent and Trademark Office.

Braly, G. W. (n.d.). Back to the future: The Wright Aeronautical Division $(W A D)$ recommended leaning procedure. General Aviation Modifications, Inc. Retrieved from http://gami.com/articles/bttf.php

Chrisman, C. G. (2011). Comparison of best economy and best power mixture settings in the Purdue University training fleet (College of Technology Directed Projects). Purdue ePubs. http://docs.lib.purdue. edu/techdirproj/42/

Cirrus Design (2008). Airplane information manual for the Cirrus Design SR20 (Report No. 13999-004). Duluth, MN: Cirrus Design.

Crane, D. (2005). Powerplant (2nd ed.). Newcastle, WA: Aviation Supplies \& Academics, Inc.

Curtiss-Wright Corporation (1957, Oct.). Basic theory of operation: Turbo compound engine. Wood-Ridge, NJ: Wright Aeronautical Division, Curtiss-Wright Corporation. (Reprinted and available from General Aviation Modifications, Inc (GAMI), see http://www.advancedpilot. com/store.html)

Eclipse Foundation (2012). Eclipse IDE for Java developers. Ottawa, Ontario: The Eclipse Foundation. Software (version Indigo Service Release 2) retrieved from http://www.eclipse.org/downloads
Garmin International, Inc. (2009). Cirrus Perspective by Garmin: Pilot's guide for the SR20/SR22 (Report 190-00820-03 Rev. A). Olathe, KS: Garmin International, Inc.

Haines, T. B. (2010, June 14). Flying lean of peak: Such heresy! AOPA Pilot Online Blog. Retrieved from http://blog.aopa.org/blog/?p=1195

Horne, T. A., \& Hirschman, D. (2011). Dogfight: Running lean of peak. AOPA Online, 54(5). Retrieved from http://www.aopa.org/members/ files/pilot/2011/may/feature_dogfight_running_lean_of_peak.html

Hirschman, D. (2009). Frugal flyer: So wrong for so long. AOPA Online, 7(52). Retrieved from http://www.aopa.org/members/files/pilot/2009/ july/frugal0907.html

Kroes, M. J., \& Wild, T. W. (1995). Aircraft powerplants (11th ed.). New York, NY: Glencoe/McGraw-Hill.

Department of Aviation Technology. (2011). Flight operations handbook (Rev. 7/11). West Lafayette, IN: Purdue University Department of Aviation Technology.

R Development Core Team (2012). R: A language and environment for statistical computing. Vienna, Austria: R Foundation for Statistical Computing. R software (version 2.15.0) retrieved from http://R-project. org

Ratcliffe, C. P., \& Rogers, D. F. (2002). Balanced fuel-injector effects on in-flight aircraft engine vibration. Journal of Aircraft, 39(3), 440-444. http://dx.doi.org/10.2514/2.2970

Sequoia Aircraft Corporation. (1990). Benchmark: Aircraft performance and testing for single- and multi-engine aircraft using constant-speed propellers. Retrieved from www.seqair.com/benchmark/Benchmark Manual.pdf

Smith, H. C. (1992). The illustrated guide to aerodynamics (2nd ed.). New York, NY: TAB Books/McGraw-Hill.

Stone, R. (1993). Introduction to internal combustion engines (2nd ed.). Warrendale, PA: Society of Automotive Engineers.

Teledyne Continental Motors (1994, Jan.). Model IO-360 series maintenance and operator's manual (Form X30617). Mobile, AL: TCM. 